\title{
A BI-DIRECTIONAL ELECTROCHEMICALLY DRIVEN MICRO LIQUID DOSING SYSTEM WITH INTEGRATED SENSOR/ACTUATOR ELECTRODES
}

\author{
Sebastian Böhm, Wouter Olthuis, Piet Bergveld \\ $M E S A^{+}$Research Institute, University of Twente, \\ P.O. Box 217, 7500 AE Enschede, The Netherlands \\ Tel: +31 534892724,Fax: +31 534892287,e-mail: s.bohm@el.utwente.nl
}

\begin{abstract}
In this contribution a micro liquid dosing system is presented, which allows bi-directional manipulation of fluids (i.e. pushing out and pulling in of liquids) by the electrochemical generation and removal of gas bubbles. Bi-directionality is obtained by reversal of the actuation current thereby causing the earlier produced gasses to react back to water. This reduction of gas volume actively pulls liquid back into the system. The electrochemical actuator electrodes have been specially designed to perform the simultaneous measurement of conductivity, via which the total amount of gas can be estimated. As this amount equals the total dosed volume of liquid, dispensed volumes can be determined on-line during experiments.
\end{abstract}

\section{INTRODUCTION}

The work described in this contribution, is part of the development of a miniaturized chemical analysis system for the on-line determination of a number of (bio)chemical substances in critically ill patients. This micro Total Analysis System ( $\mu$ TAS) $[1,2]$ has all components on board to perform a complete (bio)chemical analysis on a sample coming from a microdialysis probe implanted in the subcutaneous tissue of the patient. Analytes of interest are glucose, lactate, $\mathrm{pH}$ and electrolytes such as sodium and potassium. For the accurate measurement of these substances, micro-fabricated chemical sensors will be applied because this type of sensors can be combined with the low sample flow rate associated with microdialysis $[3,4]$. To be even more compatible with the microdialysis in-vivo sampling technique, flow through sensors are under development which are based on semi-permeable fibers also applied in the probe assembly [5]. However as all sensors show some baseline drift as well as changes in sensitivity, a calibration facility is required to provide the sensors with calibration and re-conditioning liquids prior to measurements.

For this purpose a number of systems for fluid dosing in microsystems are available. One possibility is a micro pump, integrated with the microfluidic system [6]. In this system, a flow sensor is integrated with the silicon micropump. Another possibility is the hybrid combination of a plastic micropump [7]. However, both options impose difficulties in the integration step. The process for manufacturing the former dosing system, consists of a large number of steps which makes the integration with other fluidic structures not favorable in the present case. The second pump, in this case made from plastic by conventional injection molding, has pump rates in the $\mathrm{ml} / \mathrm{min}$ range, which is too large for the accurate dosing of (sub)microliter volumes.

Therefore a simple but effective concept for dosing small, (sub)microliter amounts of liquid is to be selected, which can readily be integrated with the chemical micro system under development. An interesting actuation principle is the electrochemical generation of gas bubbles in a reservoir at noble metal electrodes. The remarkably simple construction of these types of actuators, consisting of two platinum electrodes placed in an electrolyte solution, allows easy integration and operation. This type of actuator has been applied to pressurize a closed reservoir to move a valve closing membrane [8] and in a micromachined valve [9]. In both applications no liquid is dosed but a closed liquid reservoir is pressurized. However by making an opening in this reservoir, liquid can be dosed at isobaric conditions. An example is a drug delivery system in which a constant current gives rise to a constant flow rate of a liquid drug from a separate reservoir [10]. Here the bubble reservoir, in which gas bubbles are generated, is in hydraulic contact with the reservoir containing the drug, in order to exclude gas bubbles in the drug and prevent contamination by the species generated at the electrodes. The reported dosing accuracy is about $2 \%$. 
For the purpose of integration a similar system is designed in silicon. It basically consists of a silicon fluidic board in which the various channels and reservoirs are etched. On top of this structure, a Pyrex $\otimes$ cover is bonded on which the various electrodes are patterned. First results on the dosing characteristics have been reported [11]. Further experiments on this dosing system indicated that in certain situations, inaccuracies in dosing occur. For instance due to the catalyzing action of the platinum electrodes, the mixture of hydrogen and oxygen gas will react back to water giving rise to erroneous dosed volumes. Also the influence of temperature can not always be neglected, especially if the total generated gas volume is relatively large with respect to the volume to be dosed. These effects can result in incorrect dosing if only the total injected electrical charge is applied for control.

In order to compensate for these effects, a sensor is required to determine the total gas volume in the reservoir, which is equal to the total volume dosed. In this contribution the adaptation of the actuator electrodes to enable the simultaneous measurement of the conductivity of the electrolyte/gas mixture in the bubble reservoir is described. It will be shown that this measured conductivity is related to the amount of gas in the bubble reservoir, which equals the amount of liquid dosed.

\section{THEORY}

The proposed system (see figure 1) mainly consists of a bubble reservoir with noble metal electrodes and is filled with a suitable electrolyte for bubble generation. This reservoir is in hydraulic contact with the reservoir containing the liquid to be dispensed. This container is in turn connected to the microfluidic system to which the liquid is to be delivered. current source

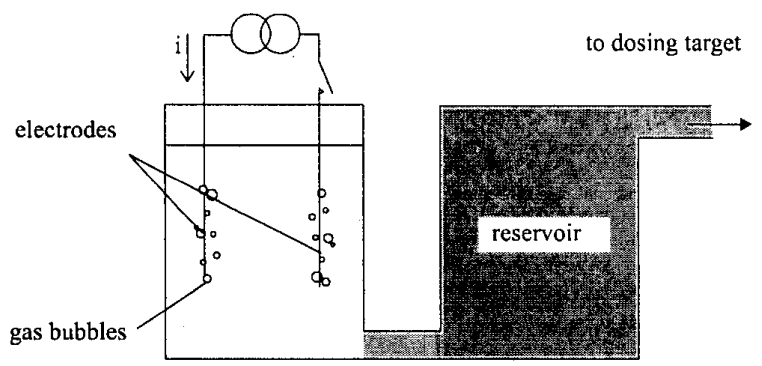

Figure 1: Proposed electrochemically driven dosing system.

The conversion of this configuration into a generic micromachined version is straightforward (see figure 2). The bubble reservoir is formed by a rectangular etched section whilst the liquid reservoir is in the form of a meander channel. The required electrodes are prepared by patterning a sputtered platinum layer on a Pyrex $(\mathbb{B}$ wafer.

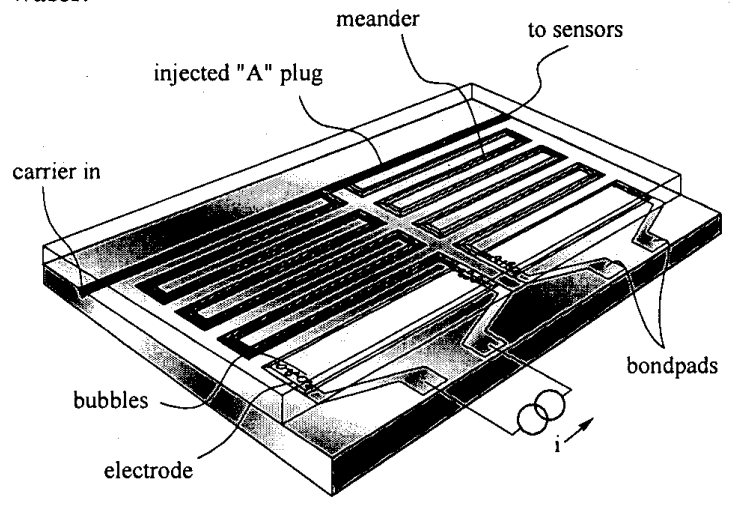

Figure 2: Implementation of a (dual) dosing system by micromachining

The chosen coulometric gas generating reaction is the well-known electrolysis reaction, occurring if an actuation current is sent through two noble metal electrodes placed in water. It then can be derived that the total amount $\Delta \mathrm{V}$ of gas that is produced by the application of a current pulse of amplitude $i$ of duration $\Delta \mathrm{t}$, is given by:

$$
\Delta V=\Delta t \frac{3}{4} \frac{i}{F} V_{m}
$$

It follows from this equation that the amount of generated gas is linearly depending on the electric charge that is injected via the actuation current. This linear relation indicates the capability of dosing by the application of this type of actuator.

In order to be able to apply the electrodes both as actuator and as conductivity sensor, interdigitated electrodes were designed and realized (see figure 3). By connecting the electrodes as indicated in this figure, the conductivity in the right cell can be measured simultaneously with the application of the actuation current and eliminates the use of switches. It should be noted that the bubble reservoir in which hydrogen is generated is twice as large as the oxygen bubble reservoir while hydrogen is generated at twice a higher rate (see reaction (1)).

The void fraction $\varepsilon$ in the bubble reservoir is represented by

$$
\varepsilon=\frac{V_{\text {gas }}}{V_{\text {reservoir }}}
$$


By this definition $\varepsilon$ is 0 before any gas bubbles are generated and can in theory increase to unity, which represents the case of a bubble reservoir completely filled with gas bubbles. If it is assumed that the penetration depth of the electric field lines originating from the interdigitated conductivity electrodes is equal to or larger than the depth of the bubble reservoir, and that the sensitivity of the conductivity sensor is homogeneous over the volume of the reservoir, the measured conductivity can generally be written as

$$
G=G_{0} \frac{1}{1-\varepsilon}
$$

where $G_{0}$ is the measured conductivity in the particular bubble reservoir at $t=0$ i.e. before any bubbles are generated.

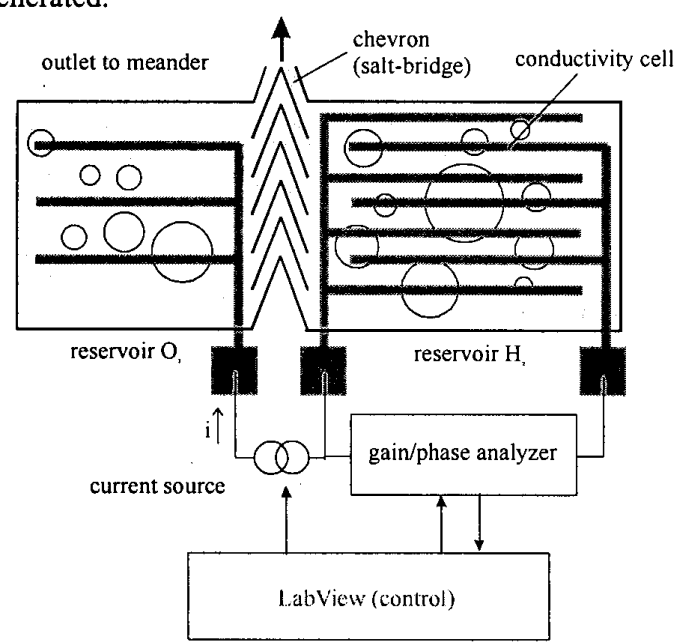

Figure 3: Cell geometry and electrical connection.

\section{EXPERIMENTAL}

The channel structures $(100 \mu \mathrm{m}$ deep) were realized by Dry Reactive Ion Etching, whilst the platinum electrode structure was formed by a lift-off process on a Pyrex $\mathbb{B}$ glass wafer. After sawing the silicon and glass wafers, individual devices were prepared by anodic bonding. A photograph of a realized device is shown in figure 4 .

The internal resistance of the interdigitated electrodes was measured to be $22 \mathrm{Ohm}$. Impedance measurements are corrected by subtracting this constant internal resistance from the measured impedance.

To ensure that only the electrolysis reactions occur, pure water was used and to provide sufficient conductivity, $500 \mathrm{mM} \mathrm{KNO} \mathrm{KN}_{3}$ was added as an inert background electrolyte. The devices were filled by placing them in a beaker filled with the electrolyte solution and then transferring this beaker to a vacuum system. After evacuation and re-aeration of this system, the air in the immersed device is replaced with electrolyte.

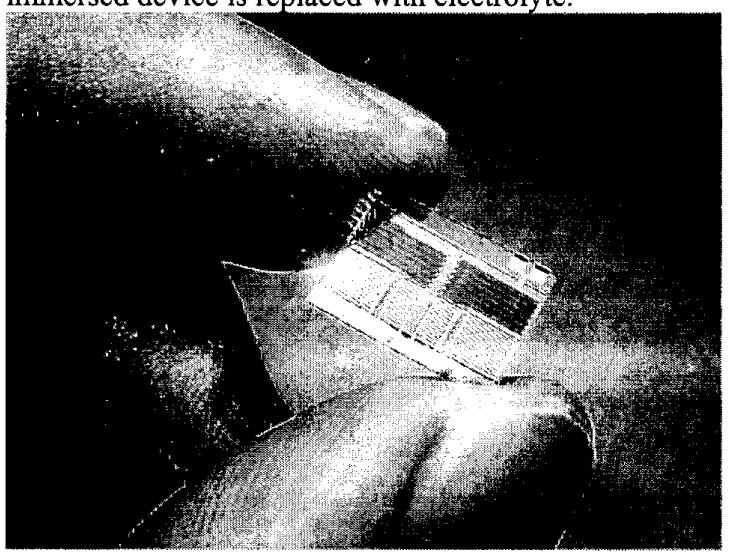

Figure 4: Photograph of the realized device.

First the working of the interdigitated electrodes as dosing sensor was evaluated by measuring the impedance at a fixed frequency of $50 \mathrm{kHz}$ (see Figure 5 ). The gas volume was measured by using a video microscope.

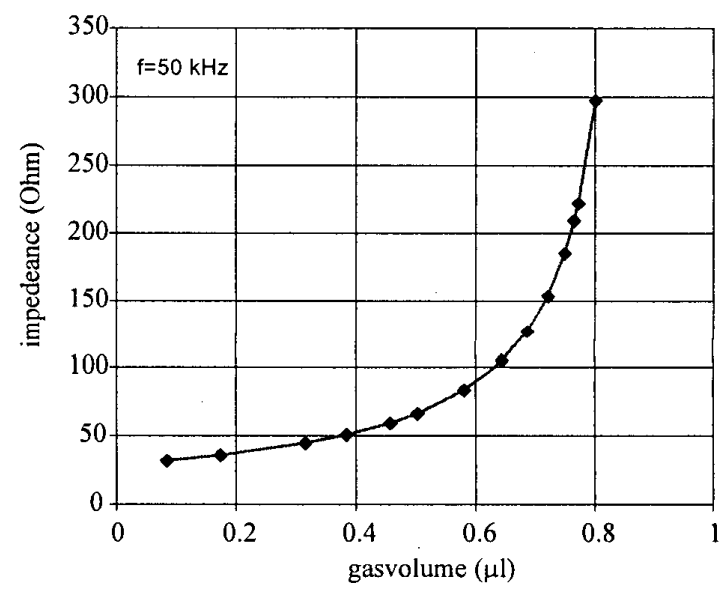

Figure 5: Measured impedance as function of dosed volume (the solid line represents theory).

As the hydrogen and oxygen gas is produced in different reservoirs, by reversal of the current, the electrolysis reaction can be reversed thereby effectively reducing the amount of previously generated gas. As a consequence, liquid can be pulled back into the system.

This process of reversing the direction of the current is illustrated in Figure 6. It can be seen from the measured conductivity, which represents the gas volume in the reservoir, that by the application of a constant reversed current gasses are reacted back to water thereby actively 
pulling liquid back in the meander. This bi-directional manipulation of liquids is highly advantageous in a number of applications, for instance to introduce sample into a (chemical) micro analysis system.

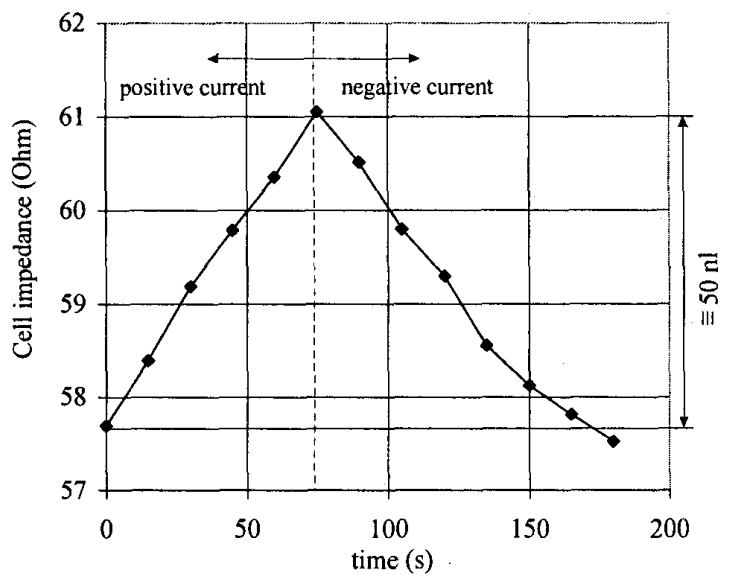

Figure 6: Drawing dispensed liquid back into the meander by current reversal. In the first phase $(t<70 \mathrm{~s})$ $50 \mathrm{nl}$ is dispensed and drawn back in the second phase.

\section{CONCLUSIONS}

An electrochemically driven dosing system, based on the generation of gas bubbles by electrolysis of water was realized. By shaping the actuator electrodes, at which the gas bubbles evolve, into an interdigitated structure the impedance of the bubble reservoir could be monitored simultaneously with actuation. It has been demonstrated that this cell impedance is a function of the total gas bubble volume in the reservoir, which equals the total volume dosed. By monitoring this cell impedance, which can easily be performed by a simple electronic circuit, the dosed volume could be determined within a few tens of $\mathrm{nl}$, and can be applied to result in an improved control of the dispensed volumes. It was also demonstrated that by reversal of the actuation current, liquid can actively be drawn back into the fluidic system.

\section{ACKNOWLEDGMENTS}

The authors whish to thank Mr. J. Bomer for the manufacturing of the devices and Mr. B. Timmer for performing measurements. This work has been financially supported by the Dutch foundation for Fundamental Research on Matter (FOM).

\section{REFERENCES}

[1] J. H. Fluitman, A. van den Berg and T. Lammerink, Micromechanical components for $\mu \mathrm{TAS}$, in proceedings of Micro Total Analysis Systems 1994, (Kluwer Academic Publishers, Dordrecht, 1994) p. 73-83.

[2] A. van den Berg, T.S.J. Lammerink, 'Micro Total Analysis Systems: microfluidic aspects, integration concepts and applications', Topics in Current Chemistry, 194, p. 21-49 (1998).

[3] J. Kehr, 'A survey on quantitative microdialysis: theoretical models and practical implications', J. Neurosc. Meth. 48, p. 251-261 (1993)

[4] P. F. Morrison, P. M. Bungay, J. K. Hsiao, I. N. Dykstra, and R. L. Dedrick (Elsevier, Amsterdam 1991), in Techniques in the Behavioral and Neural Sciences in Microdialysis in the Neurosciences 7 , (Elsevier, Amsterdam 1991) p. 47-80

[5] P. Bergveld, S. Böhm and W. Olthuis, 'Microdialysis-probe integrated with Si-chip', International Patent Application, PCT/NL99/00057, (1999).

[6] M. T. S. Elwenspoek,. T. Lammerink, R. Miyake and J. H. J. Fluitman, 'Towards integrated microliquid handling systems', J. Micromech. Microeng 4, p. 227-245 (1994).

[7] S. Böhm, W. Olthuis and P. Bergveld, 'A plastic micropump constructed with conventional techniques and materials', Accepted for publication in Sens. and Act. A.

[8] C. Neagu, J. G. E. Gardeniers, M. Elwenspoek and J. J. Kelly, 'An electrochemical microactuator: principle and first results', J. Microelectromechanical Syst. 5 (1), p. 2-9. (1996).

[9] D. A. Hopkins Jr., Electrochemical actuator and method of making same, U.S. Patent nr. 5,671,905, (1997).

[10] D. O'Keefe, C. O'Herlihy, Y. Gross and J. G. Kelly, 'Patient-controlled analgesia using a miniature electrochemically driven infusion pump', British J. Anaesthesia 73, p. 843-846 (1994).

[11] S. Böhm, W. Olthuis and P. Bergveld, 'An integrated micromachined electrochemical pump and dosing system', J. Biom. Microdev. 1:2, p. 121-130, (1999). 